PROCEEDINGS OF THE

AMERICAN MATHEMATICAL SOCIETY

Volume 137, Number 1, January 2009, Pages 195-204

S 0002-9939(08)09555-5

Article electronically published on August 7, 2008

\title{
ON THE EXPECTED NUMBER OF ZEROS OF A RANDOM HARMONIC POLYNOMIAL
}

\author{
WENBO V. LI AND ANG WEI
}

(Communicated by Michael T. Lacey)

\begin{abstract}
We study the distribution of complex zeros of Gaussian harmonic polynomials with independent complex coefficients. The expected number of zeros is evaluated by applying a formula of independent interest for the expected absolute value of quadratic forms of Gaussian random variables.
\end{abstract}

\section{INTRODUCTION}

A harmonic polynomial is a complex-valued harmonic function in $\mathbb{C}$ (the complex plane) of the form $h_{n, m}(z):=p_{n}(z)+q_{m}(\bar{z})$, where $p_{n}(z)$ and $q_{m}(z)$ are analytic polynomials of degree $n$ and $m$, respectively, with $0 \leq m \leq n$. In 1992, T. SheilSmall conjectured that the sharp upper bound for the number of zeros of $h_{n, m}(z)$ was $n^{2}$. Wilmshurst proved this in Wi98 by using Bézout's theorem and also demonstrated by examples that the bound is sharp for $m=n$ and $m=n-1$. In addition, for $m \leq n-1$, it was conjectured in Wi98 that the the maximal number of zeros should be $m(m-1)+3 n-2$. The case $m=1$ was proved in Khavinson and Swiatek KS03 by using powerful techniques from complex dynamics. Other related works can be found in BHS95, BL04, Ge03, and KN06]. Due to the variability of the number of zeros for $h_{n, m}(z)$, unlike polynomials of fixed degree, it is natural to ask for the expected number of zeros on $\mathbb{C}$ with random coefficients.

There is a long history of studying zeros of a random polynomial whose coefficients are independent, non-degenerate random variables; see EK95 for a survey. Exact formulae for the expected number of real zeros under independent identically distributed Gaussian coefficients are found for a random polynomial by Kac Kac43, and for a random trigonometric polynomial by Dunnage D66. Much work was also done on complex roots over a fixed domain; see [SV95], IZ97] and PV05. For harmonic homogeneous polynomials of degree $d$ in $m+1$ variables, that is, the sum of powers of all $m+1$ variables in each term is always $d$ and the Laplacians of the polynomials are equal to zero, the expected number of roots for a system of $m$ such random harmonic homogeneous polynomial equations is $(d(d+m-1) / m)^{m / 2}$; see EK95. One may also consider systems of homogeneous harmonic polynomials of different degrees, or one may consider underdetermined systems, and obvious generalizations of the above result still hold; see Kostlan Ko02 for a detailed discussion.

Received by the editors December 14, 2007.

2000 Mathematics Subject Classification. Primary 34F05, 60G15; Secondary 26C10.

The first author was partially supported by an NSF grant DMS-0505805.

(C)2008 American Mathematical Society 195

Reverts to public domain 28 years from publication 
In this paper, we consider a challenging general case and find the expected number of zeros in an open domain $T \subset \mathbb{C}$ for a random harmonic polynomial

$$
h_{n, m}(z)=\sum_{j=0}^{n} a_{j} z^{j}+\sum_{j=0}^{m} b_{j} \bar{z}^{j}
$$

with $0 \leq m \leq n$, where $a_{j}$ and $b_{j}$ are centered complex Gaussian random variables, i.e. $\mathbb{E} a_{j}=\mathbb{E} b_{j}=0$, with $\mathbb{E} a_{j} \bar{a}_{k}=\delta_{j k}\left(\begin{array}{c}n \\ j\end{array}\right)$ and $\mathbb{E} b_{j} \bar{b}_{k}=\delta_{j k}\left(\begin{array}{c}m \\ j\end{array}\right)$. Our argument also works for independent identically distributed standard complex or real Gaussian variables. The use of the Gaussian distribution is mainly due to feasibility of computation and provides a reasonable understanding of universality behavior under other distributions. The main theorem of this paper is:

Theorem 1.1. The expected number of zeros of $h_{n, m}(z)=p_{n}(z)+q_{m}(\bar{z})$ on an open domain $T \subset \mathbb{C}$, denoted by $\mathbb{E} N_{h}(T)$, is given by:

$$
\mathbb{E} N_{h}(T)=\frac{1}{\pi} \int_{T} \frac{1}{|z|} \frac{r_{1}^{2}+r_{2}^{2}-2 r_{12}^{2}}{r_{3}^{2} \sqrt{\left(r_{1}+r_{2}\right)^{2}-4 r_{12}^{2}}} d \sigma(z)
$$

where $\sigma(\cdot)$ denotes the Lebesgue measure on the complex plane and

$$
\begin{aligned}
& r_{12}=m n|z|^{4}\left(1+|z|^{2}\right)^{m+n-2}, r_{3}=\left(1+|z|^{2}\right)^{m}+\left(1+|z|^{2}\right)^{n}, \\
& r_{1}=r_{3}\left(1+|z|^{2}\right)^{n-2}\left(n^{2}|z|^{4}+n|z|^{2}\right)-n^{2}|z|^{4}\left(1+|z|^{2}\right)^{2 n-2}, \\
& r_{2}=r_{3}\left(1+|z|^{2}\right)^{m-2}\left(m^{2}|z|^{4}+m|z|^{2}\right)-m^{2}|z|^{4}\left(1+|z|^{2}\right)^{2 m-2} .
\end{aligned}
$$

In particular, when $m=n$, the expected number of zeros over the entire $\mathbb{C}$ is

$$
\begin{aligned}
\mathbb{E} N_{h}(\mathbb{C}) & =\frac{\pi n^{2}}{4 \sqrt{n-1}}+\frac{n}{2}-\frac{n^{2}}{2 \sqrt{n-1}} \arctan \left(\frac{1}{\sqrt{n-1}}\right) \\
& \sim \frac{\pi}{4} n^{3 / 2} \quad \text { as } \quad n \rightarrow \infty,
\end{aligned}
$$

and when $m=\alpha n+o(n)$ with $0 \leq \alpha<1, \mathbb{E} N_{h}(\mathbb{C}) \sim n$ as $n \rightarrow \infty$.

The argument principle immediately shows that a harmonic polynomial $h_{n, m}(z)$ has at least $n$ zeros. It is surprising to see that even when $m$ is a fraction of $n$, the expected number of zeros is still $n$ asymptotically. On the other hand, when $m$ is $n$ minus a constant, the expected number of zeros is of order $n^{3 / 2}$. Note that the finiteness of $\mathbb{E} N_{h}(\mathbb{C})$ shows that $h_{n, m}(z)$ has finite number of zeros with probability one. We can also prove $\mathbb{E} N_{h}(\mathbb{C})<\infty$ directly by applying Bézout's theorem, which implies that $h_{n, m}(z)=p_{n}(z)+q_{m}(\bar{z})$ has at most $n^{2}$ zeros if $p_{n}(z)$ and $q_{m}(z)$ are relatively prime; see DHL96, Wi98 and S02. Following a standard argument similar to the proof of Lemma 5 in CW03, one can show that the probability $\mathbb{P}\left(\exists z_{0} \in \mathbb{C}\right.$ s.t. $\left.p_{n}\left(z_{0}\right)=q_{m}\left(z_{0}\right)=0\right)$ is zero, which is equivalent to saying that $p_{n}(z)$ and $q_{m}(z)$ are relatively prime polynomials almost surely.

Our approach follows the general framework used in [IZ97, but significantly different arguments at the technical level are needed. To be more precise, it is crucial for us to find an expectation of the form $\mathbb{E}\left|X_{1}^{2}-X_{2}^{2}+X_{3}^{2}-X_{4}^{2}\right|$ for joint Gaussian vector $\left(X_{1}, X_{2}, X_{3}, X_{4}\right)$. In all known early work on random functions, one only needs to deal with $\mathbb{E}\left|X_{1}^{2}+X_{2}^{2}\right|=\mathbb{E} X_{1}^{2}+\mathbb{E} X_{2}^{2}$. This is why we have a very hard time finding the variance or higher moments of the number of zeros, since we do not know how to evaluate expressions like $\mathbb{E} \prod_{j=1}^{d}\left|X_{1 j}^{2}-X_{2 j}^{2}+X_{3 j}^{2}-X_{4 j}^{2}\right|$. 
Note that the difference of squares comes from the combined effect of variables $z$ and $\bar{z}$.

Our method also works in principle for the rational harmonic function, and hence provides a probabilistic interpretation of the Khavinson-Neumann theorem on the gravitationally lensed images of a light source by $n$ masses; see [KN06. Technical details will be given elsewhere.

The remaining sections are organized as follows: Section 2 deals with the evaluation of the absolute value of a quadratic form of Gaussian random variables. Our method is based on a representation of the absolute function. A useful corollary is given; for related topics of independent interest, see details in [LW08.

The main part of section 3 is devoted to the detailed proof of Theorem 1.1, followed by interesting asymptotic results. In the last section we discuss the alternative setting of random harmonic polynomials with independent identically distributed standard complex Gaussian coefficients. Finally, it must be mentioned that the first author's attention was drawn to the problem by an excellent lecture given by D. Khavinson.

\section{Preliminary Results}

We start with the Rice formula, which provides a representation for the expected number of zeros of certain random fields; see AW05 and AW06 for details.

Lemma 2.1. Let $f: U \rightarrow \mathbb{R}^{d}$ be a random field, with $U$ an open subset of $\mathbb{R}^{d}$. Assume that

(1) $f$ is Gaussian,

(2) almost surely the function $t \rightarrow h(t)$ is of class $C^{1}$,

(3) for each $t \in U, f(t)$ has a non-degenerate distribution (i.e. $\operatorname{Var}(f(t)) \succ 0)$,

(4) $\mathbb{P}\left\{\exists t \in U\right.$ s.t. $\left.f(t)=0, \operatorname{det}\left(f^{\prime}(t)\right)=0\right\}=0$.

Then, for every Borel set $T$ contained in $U$, we have

$$
\mathbb{E}\left(N_{f}(T)\right)=\int_{T} \mathbb{E}\left(\mid \operatorname{det}\left(f^{\prime}(t)\right) \| f(t)=0\right) p_{0} d t
$$

where $p_{0}$ is the probability density of $f(t)$ at 0 .

Note that function $f$ in the above formula is defined on $\mathbb{R}^{d}$. In our application, we need to find all zeros (real and complex) of $h_{n, m}(z)$. They are real zeros of $\Re h_{n, m}(x+i y)=0$ and $\Im h_{n, m}(x+i y)=0$ for $(x, y) \in \mathbb{R}^{2}$, or equivalently, $\Re h_{n, m}\left(r e^{i \theta}\right)=0$ and $\Im h_{n, m}\left(r e^{i \theta}\right)=0$, for $(r, \theta) \in \mathbb{R}^{+} \times[0,2 \pi) \subset \mathbb{R}^{2}$. It is easy to check the conditions in Lemma 2.1. In particular, condition (4) follows from Lemma 5 in CW03 based on the smoothness of $f$.

Next we give a formula for the expectation of the absolute value of a quadratic form $\langle X, H X\rangle$ of Gaussian random variables. The formula is of independent interest and a special case is used in the proof of the main theorem.

Proposition 2.1. For a real centered Gaussian random vector $X=\left(X_{1}, X_{2}, \cdots\right.$, $\left.X_{n}\right)$ with covariance matrix $R$ and any real symmetric matrix $H=\left(h_{i j}\right)_{n \times n}$,

$$
\begin{aligned}
\mathbb{E}|\langle X, H X\rangle| & =\mathbb{E}\left|\sum_{i, j=1}^{n} h_{i j} X_{i} X_{j}\right| \\
& =\frac{2}{\pi} \int_{0}^{\infty} \frac{1}{t^{2}}\left(1-\frac{\operatorname{det}(I+2 \mathrm{i} t R H)^{1 / 2}+\operatorname{det}(I-2 \mathrm{i} t R H)^{1 / 2}}{2 \operatorname{det}\left(I+4 t^{2} R^{2} H^{2}\right)^{1 / 2}}\right) d t
\end{aligned}
$$


where $I$ is the $n \times n$ identity matrix and $\mathbf{i}=\sqrt{-1}$.

Proof. We only need to consider a non-singular covariance matrix $R$, since for singular $R$ the result still holds by considering $R^{*}=R+\delta I$ and using the standard limiting argument of taking $\delta \rightarrow 0$.

We start with the representation

$$
|x|=\frac{2}{\pi} \int_{0}^{\infty} \frac{1-\cos (x t)}{t^{2}} d t=\frac{2}{\pi} \int_{0}^{\infty} \frac{1-\mathbb{E}_{\varepsilon} e^{\mathrm{i} \varepsilon x t}}{t^{2}} d t
$$

where $\mathbb{P}(\varepsilon=1)=\mathbb{P}(\varepsilon=-1)=1 / 2$. Then we can rewrite the expectation as

$$
\mathbb{E}|\langle X, H X\rangle|=\frac{2}{\pi} \int_{0}^{\infty} \frac{1}{t^{2}}\left(1-\mathbb{E}_{\varepsilon} \mathbb{E}_{X} e^{i \varepsilon t\langle X, H X\rangle}\right) d t .
$$

For a non-singular covariance matrix $R$, the density of $X$ is

$$
f_{X}(x)=(2 \pi)^{-n / 2}(\operatorname{det} R)^{-1 / 2} e^{-\frac{1}{2}\left\langle x, R^{-1} x\right\rangle} .
$$

Therefore we have

$$
\begin{aligned}
\mathbb{E}_{X} e^{\mathrm{i} \varepsilon t\langle X, H X\rangle} & =\int_{\mathbb{R}^{n}}(2 \pi)^{-n / 2}(\operatorname{det} R)^{-1 / 2} e^{-\frac{1}{2}\left\langle x, R^{-1} x\right\rangle} e^{\mathrm{i} \varepsilon t\langle x, H x\rangle} d x \\
& =(\operatorname{det} R)^{-1 / 2} \int_{\mathbb{R}^{n}}(2 \pi)^{-n / 2} e^{-\frac{1}{2}\left\langle x,\left(R^{-1}-2 \mathrm{i} t \varepsilon H\right) x\right\rangle} d x \\
& =(\operatorname{det} R)^{-1 / 2}\left(\operatorname{det}\left(R^{-1}-2 \mathrm{i} t \varepsilon H\right)\right)^{-1 / 2} \\
& =(\operatorname{det}(I-2 \mathrm{i} t \varepsilon R H))^{-1 / 2} .
\end{aligned}
$$

Note that

$$
\operatorname{det}(I-2 \mathrm{i} t \varepsilon R H) \cdot \operatorname{det}(I+2 \mathrm{i} t \varepsilon R H)=\operatorname{det}\left(I+4 t^{2} R^{2} H^{2}\right) \neq 0,
$$

and so $\operatorname{det}(I-2 \mathrm{i} t \varepsilon R H) \neq 0$. Hence

$$
\begin{aligned}
\mathbb{E}_{\varepsilon} \mathbb{E}_{X} e^{\mathrm{i} \varepsilon t\langle X, H X\rangle} & =\frac{1}{2} \frac{1}{\operatorname{det}(I-2 \mathrm{i} t R H)^{1 / 2}}+\frac{1}{2} \frac{1}{\operatorname{det}(I+2 \mathrm{i} t R H)^{1 / 2}} \\
& =\frac{\operatorname{det}(I-2 \mathrm{i} t R H)^{1 / 2}+\operatorname{det}(I+2 \mathrm{i} t R H)^{1 / 2}}{2 \operatorname{det}\left(I+4 t^{2} R^{2} H^{2}\right)^{1 / 2}},
\end{aligned}
$$

which is real since $\operatorname{det}(I-2 \mathrm{i} t R H)^{1 / 2}$ and $\operatorname{det}(I+2 \mathrm{i} t R H)^{1 / 2}$ are conjugate to each other. Thus we finish the proof.

It is obvious that in the case where the matrix $H=\left(h_{i j}\right)_{n \times n}$ is positive definite, we have directly

$$
\mathbb{E}|\langle X, H X\rangle|=\mathbb{E}\langle X, H X\rangle=\sum_{i, j=1}^{n} h_{i j} r_{i j}
$$

where $\left(r_{i j}\right)_{n \times n}=R$ is the covariance matrix of $X$.

Next we give an important corollary which will be used in the proof of Theorem 1.1. Other interesting cases and applications can be found in [LW08.

Corollary 2.1. Let $\left(X_{1}, X_{2}\right)$ be a centered Gaussian with $\mathbb{E} X_{1}^{2}=\sigma_{1}^{2}, \mathbb{E} X_{2}^{2}=\sigma_{2}^{2}$, and $\mathbb{E} X_{1} X_{2}=\sigma_{12}$. If $\left(X_{3}, X_{4}\right)$ is an independent copy of $\left(X_{1}, X_{2}\right)$, then

$$
\mathbb{E}\left|X_{1}^{2}-X_{2}^{2}+X_{3}^{2}-X_{4}^{2}\right|=\frac{2 \sigma_{1}^{4}+2 \sigma_{2}^{4}-4 \sigma_{12}^{2}}{\sqrt{\left(\sigma_{1}^{2}+\sigma_{2}^{2}\right)^{2}-4 \sigma_{12}^{2}}} .
$$


Proof. In this case, we have $H=\operatorname{diag}(1,-1,1,-1)$ and

$$
R=\left(\begin{array}{cccc}
\sigma_{1}^{2} & \sigma_{12} & 0 & 0 \\
\sigma_{12} & \sigma_{2}^{2} & 0 & 0 \\
0 & 0 & \sigma_{1}^{2} & \sigma_{12} \\
0 & 0 & \sigma_{12} & \sigma_{2}^{2}
\end{array}\right) .
$$

Therefore

$$
I+2 \mathrm{i} t R H=\left(\begin{array}{cccc}
1+2 \mathrm{i} t \sigma_{1}^{2} & -2 \mathrm{i} t \sigma_{12} & 0 & 0 \\
2 \mathrm{i} t \sigma_{12} & 1-2 \mathrm{i} t \sigma_{2}^{2} & 0 & 0 \\
0 & 0 & 1+2 \mathrm{i} t \sigma_{1}^{2} & -2 \mathrm{i} t \sigma_{12} \\
0 & 0 & 2 \mathrm{i} t \sigma_{12} & 1-2 \mathrm{i} t \sigma_{2}^{2}
\end{array}\right)
$$

Hence the determinant is given by

$$
\operatorname{det}(I+2 \mathrm{i} t R H)=\left(1+2\left(\sigma_{1}^{2}-\sigma_{2}^{2}\right) \mathbf{i} t+\left(4 \sigma_{1}^{2} \sigma_{2}^{2}-4 \sigma_{12}^{2}\right) t^{2}\right)^{2} .
$$

Write $p=4 \sigma_{1}^{2} \sigma_{2}^{2}-4 \sigma_{12}^{2}$ and $q=\sigma_{1}^{2}-\sigma_{2}^{2}$; then Proposition 2.1 can be simplified:

$$
\begin{aligned}
\mathbb{E}\left|X_{1}^{2}+X_{2}^{2}-X_{3}^{2}-X_{4}^{2}\right| & =\frac{2}{\pi} \int_{0}^{\infty} \frac{1}{t^{2}}\left(1-\frac{1+p t^{2}}{\left(1+p t^{2}\right)^{2}+4 q^{2} t^{2}}\right) d t \\
& =\frac{2}{\pi} \int_{0}^{\infty} \frac{p\left(1+p t^{2}\right)+4 q^{2}}{\left(1+p t^{2}\right)^{2}+4 q^{2} t^{2}} d t \\
& =\frac{1}{\pi} \int_{0}^{\infty} \frac{p+4 q^{2}-2 \mathrm{i} p q t}{p t^{2}+1-2 \mathrm{i} q t}+\frac{p+4 q^{2}+2 \mathrm{i} p q t}{p t^{2}+1-2 \mathrm{i} q t} d t \\
& =\frac{p+2 q^{2}}{\sqrt{p+q^{2}}}=\frac{2 \sigma_{1}^{4}+2 \sigma_{2}^{4}-4 \sigma_{12}^{2}}{\sqrt{\left(\sigma_{1}^{2}+\sigma_{2}^{2}\right)^{2}-4 \sigma_{12}^{2}}} .
\end{aligned}
$$

\section{Proof of Theorem 1.1}

In order to apply the representation of the number of zeros given in (2.1), we need to separate the real and imaginary parts of $h_{n, m}(z)$. Namely, we write

$$
\begin{aligned}
h_{n, m}(z) & =\sum_{j=0}^{n} a_{j} z^{j}+\sum_{j=0}^{m} b_{j} \bar{z}^{j} \\
& =\sum_{j=0}^{n}\left(a_{j, 1}+i a_{j, 2}\right) r^{j}(\cos j \theta+i \sin j \theta)+\sum_{j=0}^{m}\left(b_{j, 1}+i b_{j, 2}\right) r^{j}(\cos j \theta-i \sin j \theta) \\
& =Y_{1}(r, \theta)+i Y_{2}(r, \theta)
\end{aligned}
$$

where

$$
\begin{aligned}
& Y_{1}(r, \theta)=\sum_{j=0}^{n} r^{j}\left(a_{j, 1} \cos j \theta-a_{j, 2} \sin j \theta\right)+\sum_{j=0}^{m} r^{j}\left(b_{j, 1} \cos j \theta+b_{j, 2} \sin j \theta\right), \\
& Y_{2}(r, \theta)=\sum_{j=0}^{n} r^{j}\left(a_{j, 1} \sin j \theta+a_{j, 2} \cos j \theta\right)+\sum_{j=0}^{m} r^{j}\left(b_{j, 2} \cos j \theta-b_{j, 1} \sin j \theta\right) .
\end{aligned}
$$


Write $Y(r, \theta)=\left(Y_{1}(r, \theta), Y_{2}(r, \theta)\right)^{T}$; the Jacobian determinant in (2.1) of this vector can be computed as

$$
\begin{aligned}
& \operatorname{det} \nabla Y(r, \theta)=\frac{\partial Y_{1}(r, \theta)}{\partial r} \frac{\partial Y_{2}(r, \theta)}{\partial \theta}-\frac{\partial Y_{1}(r, \theta)}{\partial \theta} \frac{\partial Y_{2}(r, \theta)}{\partial r} \\
& =\frac{1}{r}\left\{\left(\sum_{j=1}^{n} j r^{j}\left(a_{j, 1} \cos j \theta-a_{j, 2} \sin j \theta\right)\right)^{2}-\left(\sum_{j=1}^{m} j r^{j}\left(b_{j, 1} \cos j \theta+b_{j, 2} \sin j \theta\right)\right)^{2}\right. \\
& \left.+\left(\sum_{j=1}^{n} j r^{j}\left(a_{j, 1} \sin j \theta+a_{j, 2} \cos j \theta\right)\right)^{2}-\left(\sum_{j=1}^{n} j r^{j}\left(b_{j, 2} \cos j \theta-b_{j, 1} \sin j \theta\right)\right)^{2}\right\}
\end{aligned}
$$

To reduce the number of parameters, we change the expression back to a function of $z$. We cannot do this at the beginning because the representation of the number of zeros can only be applied to real functions. Note that $\Re z^{j}=r^{j} \cos j \theta$ and $\Im z^{j}=r^{j} \sin j \theta$, so we have

$$
\operatorname{det} \nabla Y(r, \theta)=\frac{1}{|z|}\left(u_{1}^{2}-u_{2}^{2}+v_{1}^{2}-v_{2}^{2}\right)
$$

where

$$
\begin{aligned}
& u_{1}=\Re \sum_{j=1}^{n} j z^{j} a_{j}, \quad v_{1}=\Im \sum_{j=1}^{n} j z^{j} a_{j}, \\
& u_{2}=\Re \sum_{j=1}^{m} j z^{j} a_{j}, \quad v_{2}=\Im \sum_{j=1}^{m} j z^{j} a_{j} .
\end{aligned}
$$

To simplify notation, we also define

$$
u_{3}=\Re\left(p_{n}(z)+q_{m}(\bar{z})\right), \quad v_{3}=\Im\left(p_{n}(z)+q_{m}(\bar{z})\right) .
$$

Then according to (2.1), we need to find the conditional expectation

$$
\mathbb{E}\left(\left|u_{1}^{2}-u_{2}^{2}+v_{1}^{2}-v_{2}^{2}\right| \mid u_{3}=0, v_{3}=0\right)=\mathbb{E}\left|U_{1}^{2}-U_{2}^{2}+V_{1}^{2}-V_{2}^{2}\right|
$$

where $\left(U_{1}, U_{2}, V_{1}, V_{2}\right)$ is the Gaussian random vector with the same distribution as $\left(u_{1}, u_{2}, v_{1}, v_{2}\right)$ under the condition $u_{3}=0, v_{3}=0$. According to [T90, page 34] the covariance matrix of $\left(U_{1}, U_{2}, V_{1}, V_{2}\right)$ is given by

$$
R_{c}=C-B A^{-1} B^{T}
$$

where $A_{2 \times 2}=\operatorname{cov}\left(u_{3}, v_{3}\right), B_{4 \times 2}=\operatorname{cov}\left(\left(u_{1}, u_{2}, v_{1}, v_{2}\right),\left(u_{3}, v_{3}\right)\right)$ and $C_{4 \times 4}=\operatorname{cov}\left(u_{1}\right.$, $\left.u_{2}, v_{1}, v_{2}\right)$. From (3.1) and (3.2), we have

$$
\begin{aligned}
& \mathbb{E} u_{3}^{2}=\mathbb{E} v_{3}^{2}=\frac{1}{2} \sum_{j=0}^{n}\left(\begin{array}{c}
n \\
j
\end{array}\right)|z|^{2 j}+\frac{1}{2} \sum_{j=0}^{m}\left(\begin{array}{c}
m \\
j
\end{array}\right)|z|^{2 j}=\frac{1}{2}\left(1+|z|^{2}\right)^{n}+\frac{1}{2}\left(1+|z|^{2}\right)^{m}, \\
& \mathbb{E} u_{1} u_{3}=\mathbb{E} v_{1} v_{3}=\frac{1}{2} \sum_{j=1}^{n}\left(\begin{array}{c}
n \\
j
\end{array}\right) j|z|^{2 j}=\frac{1}{2} n|z|^{2}\left(1+|z|^{2}\right)^{n-1} \\
& \mathbb{E} u_{1}^{2}=\mathbb{E} v_{1}^{2}=\frac{1}{2} \sum_{j=1}^{n}\left(\begin{array}{c}
n \\
j
\end{array}\right) j^{2}|z|^{2 j}=\frac{1}{2}\left(n^{2}|z|^{4}+n|z|^{2}\right)\left(1+|z|^{2}\right)^{n-2}
\end{aligned}
$$


Similarly we also have

$$
\begin{aligned}
& \mathbb{E} u_{2} u_{3}=\mathbb{E} v_{2} v_{3}=\frac{1}{2} m|z|^{2}\left(1+|z|^{2}\right)^{m-1}, \\
& \mathbb{E} u_{2}^{2}=\mathbb{E} v_{2}^{2}=\frac{1}{2}\left(m^{2}|z|^{4}+m|z|^{2}\right)\left(1+|z|^{2}\right)^{m-2} .
\end{aligned}
$$

Thus the covariance matrices are

$$
\begin{aligned}
A_{2 \times 2}= & \frac{1}{2} \operatorname{diag}\left(\left(1+|z|^{2}\right)^{n}+\left(1+|z|^{2}\right)^{m},\left(1+|z|^{2}\right)^{n}+\left(1+|z|^{2}\right)^{m}\right), \\
B_{4 \times 2}= & \frac{1}{2}\left(\begin{array}{cc}
n|z|^{2}\left(1+|z|^{2}\right)^{n-1} & 0 \\
m|z|^{2}\left(1+|z|^{2}\right)^{m-1} & 0 \\
0 & n|z|^{2}\left(1+|z|^{2}\right)^{n-1} \\
0 & m|z|^{2}\left(1+|z|^{2}\right)^{m-1}
\end{array}\right), \\
C_{4 \times 4}= & \frac{1}{2} \operatorname{diag}\left(\left(n^{2}|z|^{4}+n|z|^{2}\right)\left(1+|z|^{2}\right)^{n-2},\left(m^{2}|z|^{4}+m|z|^{2}\right)\left(1+|z|^{2}\right)^{m-2},\right. \\
& \left.\left(n^{2}|z|^{4}+n|z|^{2}\right)\left(1+|z|^{2}\right)^{n-2},\left(m^{2}|z|^{4}+m|z|^{2}\right)\left(1+|z|^{2}\right)^{m-2}\right) .
\end{aligned}
$$

Therefore following (3.4) we obtain the covariance matrix of $\left(U_{1}, U_{2}, V_{1}, V_{2}\right)$,

$$
R_{c}=\frac{1}{2 r_{3}}\left(\begin{array}{cccc}
r_{1} & -r_{12} & 0 & 0 \\
-r_{12} & r_{2} & 0 & 0 \\
0 & 0 & r_{1} & -r_{12} \\
0 & 0 & -r_{12} & r_{2}
\end{array}\right)
$$

where $r_{1}, r_{2}, r_{12}$ and $r_{3}$ are given in Theorem 1.1.

By applying Corollary 2.1, we obtain the expectation of the absolute value in (3.3):

$$
\mathbb{E}\left|U_{1}^{2}-U_{2}^{2}+V_{1}^{2}-V_{2}^{2}\right|=\frac{1}{r_{3}} \frac{r_{1}^{2}+r_{2}^{2}-2 r_{12}^{2}}{\sqrt{\left(r_{1}+r_{2}\right)^{2}-4 r_{12}^{2}}} .
$$

Note that $p_{0}$ is the probability density of $h(z)$ at 0 , which means

$$
p_{0}=(2 \pi)^{-1}\left(\operatorname{det}\left(\mathbb{E} Y(r, \theta) Y(r, \theta)^{T}\right)\right)^{-1 / 2}=\left(\pi r_{3}\right)^{-1}
$$

where $Y(r, \theta)=\left(Y_{1}(r, \theta), Y_{2}(r, \theta)\right)^{T}$ as defined at the beginning of this section. Combining these, we have

$$
\begin{aligned}
\mathbb{E}\left(N_{h}(T)\right) & =\int_{T} \frac{1}{|z|} \mathbb{E}\left|U_{1}^{2}-U_{2}^{2}+V_{1}^{2}-V_{2}^{2}\right| p_{0} d \sigma(z) \\
& =\frac{1}{\pi} \int_{T} \frac{r_{1}^{2}+r_{2}^{2}-2 r_{12}^{2}}{|z| r_{3}^{2} \sqrt{\left(r_{1}+r_{2}\right)^{2}-4 r_{12}^{2}}} d \sigma(z) .
\end{aligned}
$$

In the case that $m=n$, we have $r_{1}=r_{2}$, and part of the integrand in (3.5) can be simplified:

$$
\frac{r_{1}^{2}+r_{2}^{2}-2 r_{12}^{2}}{r_{3}^{2} \sqrt{\left(r_{1}+r_{2}\right)^{2}-4 r_{12}^{2}}}=\frac{\sqrt{r_{1}^{2}-r_{12}^{2}}}{r_{3}^{2}}=\frac{\sqrt{n^{2}|z|^{4}+n^{3}|z|^{6}}}{2\left(1+|z|^{2}\right)^{2}}
$$


Therefore the expected number of zeros in $\mathbb{C}$ is

$$
\begin{aligned}
\mathbb{E} N_{h}(\mathbb{C}) & =\pi^{-1} \int_{0}^{2 \pi} \int_{0}^{\infty} \frac{n r \sqrt{1+n r^{2}}}{2\left(1+r^{2}\right)^{2}} d r d \theta \\
& =n^{2} \int_{1}^{\infty}\left(\frac{1}{s^{2}+n-1}-\frac{n-1}{\left(s^{2}+n-1\right)^{2}}\right) d s \\
& =\frac{\pi n^{2}}{4 \sqrt{n-1}}+\frac{n}{2}-\frac{n^{2}}{2 \sqrt{n-1}} \arctan \left(\frac{1}{\sqrt{n-1}}\right) \\
& \sim \frac{\pi}{4} n^{3 / 2}
\end{aligned}
$$

by using the substitution $s=\sqrt{1+n r^{2}}$.

In the case that $m=\alpha n+o(n)$ with $0 \leq \alpha<1$, clearly $r_{2}$ and $r_{12}$ are dominated by $r_{1}$; therefore we have

$$
\lim _{n \rightarrow \infty} \frac{1}{n} \frac{r_{1}^{2}+r_{2}^{2}-2 r_{12}^{2}}{r_{3}^{2} \sqrt{\left(r_{1}+r_{2}\right)^{2}-4 r_{12}^{2}}}=\lim _{n \rightarrow \infty} \frac{1}{n} \frac{r_{1}}{r_{3}^{2}}=\frac{|z|^{2}}{\left(1+|z|^{2}\right)^{2}} .
$$

So the asymptotic result for $\mathbb{E} N_{h}(\mathbb{C})$ is

$$
\lim _{n \rightarrow \infty} \frac{1}{n} \mathbb{E} N_{h}(\mathbb{C})=\frac{1}{\pi} \int_{\mathbb{C}} \frac{|z|^{2}}{|z|\left(1+|z|^{2}\right)^{2}} d \sigma(z)=1 .
$$

Thus we finish the proof.

For the remaining part of this section, we briefly examine the asymptotic results for $m$ close to $n$. In the case that $m=n-k$ for a fixed positive integer $k$, we have

$$
\frac{r_{1}^{2}+r_{2}^{2}-2 r_{12}^{2}}{r_{3}^{2} \sqrt{\left(r_{1}+r_{2}\right)^{2}-4 r_{12}^{2}}} \sim \frac{n^{3 / 2} r^{3}\left(1+r^{2}\right)^{k / 2-2}}{1+\left(1+r^{2}\right)^{k}} \quad \text { as } n \rightarrow \infty \text {. }
$$

Thus $\lim _{n \rightarrow \infty} \mathbb{E} N_{h}(\mathbb{C}) / n^{3 / 2}=c_{k}$, where

$$
c_{k}=\int_{0}^{\infty} \frac{2 r^{2}\left(1+r^{2}\right)^{k / 2-2}}{1+\left(1+r^{2}\right)^{k}} d r .
$$

When $k=0$, the case degenerates into $m=n$ with $c_{0}=\pi / 4$. For $k \geq 1, c_{1}=$ $0.49 \cdots, c_{2}=0.31 \cdots$, and $c_{k}$ decreases to zero as $k \rightarrow \infty$. In fact, as $k \rightarrow \infty$, $c_{k} \sim \sqrt{2 \pi} k^{-3 / 2}$.

\section{INDEPENDENT IDENTICALLY DISTRIBUted SETTING}

Next we consider another general case,

$$
\tilde{h}_{n, m}(z)=\sum_{j=0}^{n} \tilde{a}_{j} z^{j}+\sum_{j=0}^{m} \tilde{b}_{j} \bar{z}^{j}
$$

with $0 \leq m \leq n$, where $\tilde{a}_{j}$ and $\tilde{b}_{j}$ are independent identically distributed complex Gaussian random variables with $\mathbb{E} \tilde{a}_{j}=\mathbb{E} \tilde{b}_{j}=0$ and $\mathbb{E} \tilde{a}_{j} \overline{\tilde{a}}_{k}=\delta_{j k}$. In this case, the differences when compared with the setting in the previous section are the values of $r_{1}, r_{2}, r_{12}$ and $r_{3}$. So we omit the details and only state the result. 
Theorem 4.1. The expected number of zeros of $\tilde{h}_{n, m}(z)=\tilde{p}_{n}(z)+\tilde{q}_{m}(\bar{z})$ on $T$, denoted by $\mathbb{E} N_{\tilde{h}}(T)$, is given by

$$
\mathbb{E} N_{\tilde{h}}(T)=\frac{1}{\pi} \int_{T} \frac{1}{|z|} \frac{\tilde{r}_{1}^{2}+\tilde{r}_{2}^{2}-2 \tilde{r}_{12}^{2}}{\tilde{r}_{3}^{2} \sqrt{\left(\tilde{r}_{1}+\tilde{r}_{2}\right)^{2}-4 \tilde{r}_{12}^{2}}} d \sigma(z)
$$

where $\sigma(\cdot)$ denotes the Lebesgue measure on the complex plane and

$$
\begin{aligned}
& \tilde{r}_{12}=\left(\sum_{j=1}^{n} j|z|^{2 j}\right)\left(\sum_{j=1}^{m} j|z|^{2 j}\right), \quad \tilde{r}_{3}=\sum_{j=0}^{n}|z|^{2 j}+\sum_{j=0}^{m}|z|^{2 j} \\
& \tilde{r}_{1}=\tilde{r}_{3} \sum_{j=1}^{n} j^{2}|z|^{2 j}-\left(\sum_{j=1}^{n} j|z|^{2 j}\right)^{2}, \quad \tilde{r}_{2}=\tilde{r}_{3} \sum_{j=1}^{m} j^{2}|z|^{2 j}-\left(\sum_{j=1}^{m} j|z|^{2 j}\right)^{2} .
\end{aligned}
$$

Numerical analysis suggests that the asymptotic of above expectation for $T=\mathbb{C}$ is $\lim _{n \rightarrow \infty} \mathbb{E} N_{h}(\mathbb{C}) / n=1$ for fixed $m$, but a rigorous analytic asymptotic hasn't been found.

\section{REFERENCES}

[AW05] J.-M. Azaïs and M. Wschebor, On the distribution of the maximum of a Gaussian field with $d$ parameters, Ann. Appl. Probab. 15 (1A) (2005), 254-278. MR2115043 (2005k:60117)

[AW06] J.-M. Azaïs and M. Wschebor, A self contained proof of the Rice formula for random fields, (2006), preprint available at: http://www.lsp.ups-tsle.fr/ Azais/publi/completeproof.pdf .

[BD97] P. Bleher and X. Di, Correlations between zeros of a random polynomial, J. Statist. Phys. 88 (1997), no. 1-2, 269-305. MR.1468385 (98m:60078)

[BHS95] D. Bshouty, W. Hengartner and T. Suez, The exact bound of the number of zeros of harmonic polynomials, J. d'Analyse Math. 67 (1995), 207-218. MR:1383494 (97f:30025)

[BL04] D. Bshouty and A. Lyzzaik, On Crofoot-Sarason's conjecture for harmonic polynomials, Comput. Methods Funct. Theory 4 (2004), 35-41. MR2081663 (2005e:30008)

[CW03] F. Cucker and M. Wschebor, On the expected condition number of linear programming problems, Numer. Math. 94 (2003), 419-478. MR1981163(2005m:90172)

[D66] J.E.A. Dunnage, The number of real zeros of a random trigonometric polynomial, Proc. London Math. Soc. (3) 16 (1966), 53-84. MR0192532(33:757)

[DHL96] P. Duren, W. Hengartner and R.S. Laugesen, The argument principle for harmonic functions, Amer. Math. Monthly 103 (1996), 411-415. MR.1400723 (97f:30002)

[EK95] A. Edelman and E. Kostlan, How many zeros of a random polynomial are real?, Bull. Amer. Math. Soc. 32 (1995), 1-37. MR1290398(95m:60082)

[Ge03] L. Geyer, Sharp bounds for the valence of certain harmonic polynomials, Proc. Amer. Math. Soc. 136 (2003), no. 2, 549-555. MR2358495

[IZ97] I.Ibragimov and O. Zeitouni, On roots of random polynomials, Trans. Amer. Math. Soc. 349 (1997), no. 6, 2427-2441. MR.1390040 (97h:60050)

[Kac43] M. Kac, On the average number of real roots of a random algebraic equation. Bull. Amer. Math. Soc. 49 (1943), 314-320. MR0007812(4:196d)

[KN06] D. Khavinson and G. Neumann, On the number of zeros of certain rational harmonic functions, Proc. Amer. Math. Soc. 134 (2006), 1077-1085. MR2196041 (2007h:26018)

[KS03] D. Khavinson and G. Swiatek, On the number of zeros of certain harmonic polynomials, Proc. Amer. Math. Soc. 131 (2003), 409-414. MR1933331 (2003j:30015)

[Ko02] E. Kostlan, On the expected number of real roots of a system of random polynomial equations, Foundations of computational mathematics, 149-188, World Sci. Publishing, River Edge, NJ, 2002. MR2021981 (2004k:60150)

[LW08] W.V. Li and A. Wei, Gaussian integrals for absolute value functions, in preparation.

[PV05] Y. Peres and B. Virag, Zeros of the iid Gaussian power series: A conformally invariant determinantal process, Acta Math. 194 (2005), no. 1, 1-35. MR2231337 (2007m:60150) 
[S02] T. Sheil-Small, Complex polynomials, Cambridge Studies in Advanced Mathematics 75, Cambridge University Press, Cambridge, 2002. MR1962935 (2004b:30001)

[SV95] L. Shepp and R. Vanderbei, The complex zeros of random polynomials, Trans. Amer. Math. Soc. 347 (1995), no. 11, 4365-4384. MR.1308023 (96a:30006)

[T90] Y.L. Tong, The multivariate normal distribution, Springer-Verlag, 1990. MR 1029032 (91g:60021)

[Wi98] A.S. Wilmshurst, The valence of harmonic polynomials, Proc. Amer. Math. Soc. 126 (1998), 2077-2081. MR1443416 (98h:30029)

Department of Mathematical Sciences, 517B Ewing Hall, University of Delaware, Newark, Delaware 19716

E-mail address: wli@math.udel.edu

Department of Mathematical Sciences, 308 Ewing Hall, University of Delaware, Newark, Delaware 19716

E-mail address: wei@math.udel.edu 